\title{
OPTIMIZED HEAT INTERCEPTION FOR CRYOGEN TANK SUPPORT
}

\author{
E. R. Canavan and F. K. Miller \\ Code 552 - Cryogenics and Fluids Branch \\ NASA - Goddard Space Flight Center \\ Greenbelt, Maryland, 20771, USA
}

\begin{abstract}
We consider means for using the cooling available in boil-off gas to intercept heat conducted through the support structure of a cryogen tank. A one-dimensional model of the structure coupled to a gas stream gives an analytical expression for heat leak in terms of flow rate for temperature independent-properties and laminar flow. A numerical model has been developed for heat transfer on a thin cylindrical tube with an attached vent line. The model is used to determine the vent path layout that will minimize heat flow into the cryogen tank. The results are useful for a number of applications, but the one of interest in this study is the minimization of the boil-off in large cryopropellant tanks in low Earth and low lunar orbit.
\end{abstract}

KEYWORDS: cryogenics; tank support; heat interception

\section{INTRODUCTION}

Optimizing heat interception in cryogen storage systems is an old problem in cryogenic engineering, and there have been many important advances in this area. Bejan and Smith [1] applied entropy generation minimization to the problem of cooling a structural support with gas streams. Bejan [2] extended this result to the case of discrete cooling points along the support. Chato and Khodadi [3] considered a system with both structural supports and cooled shields. More recently, Hofmann [4] derived a solution for the number and placement of shields cooled by boil-off gas.

These optimizations however, are generally applicable to ground-based cryostats. Cryogens tanks for space have the additional requirement that the overall system mass be minimized. Thus, the mass of any additional hardware for reducing the heat leak, such as cooled shields, must be weighed against the mass of simply making tanks large enough to achieve the required lifetime with a higher boil-off. Shields, must withstand transmitted vibration and acoustic loads, and must meet minimum mode frequency requirements. This 
' causes the thickness, or mass per unit area, to increase with scale. Thus, shields become less attractive at large scales. They are also less beneficial for fluids with higher heat of vaporization. However, use of boil-off gas to cool tank structural supports has little mass penalty and appears to be attractive at any scale.

Here we consider the design of a practical heat exchanger for cooling the support structure, chiefly for large tanks. High heat transfer between the gas and the support is implicit in other work on optimized cooled supports. However, in a spaceflight tank, especially cryopropellant tanks that have no vacuum shell, it is likely that the vent tube will need to be sized to carry the initial, high heat load that the tank will experience as it reaches orbit. Thus, as the tank reaches steady state, the flow velocity in the tube will likely be well below the critical Reynolds number, so the dependence on the heat transfer coefficient needs to be examined.

\section{ONE DIMENSIONAL ANALYSIS}

The one-dimensional case provides valuable insight into the problem, because under the assumption of temperature-independent property values, it yields an analytic solution. Figure 1 shows a schematic of the geometry. A tube carrying cold gas flows parallel to the support. If the thickness of the support is small or if its conductance is high in the direction normal to the flow, it will be isothermal in planes normal to the flow, and the temperature of the support $T_{s}$, will be a function only of the distance along the flow direction, $x$. Assuming constant thermal conductivity, $k_{s}$, and cross sectional area, $A$, the energy balance for the support is

$$
k_{s} A \frac{d^{2} T_{s}}{d x^{2}}=h P\left(T_{s}-T_{f}\right)
$$

where $h$ is the heat transfer coefficient, $P$ is the tube perimeter, and $T_{f}$ is the fluid

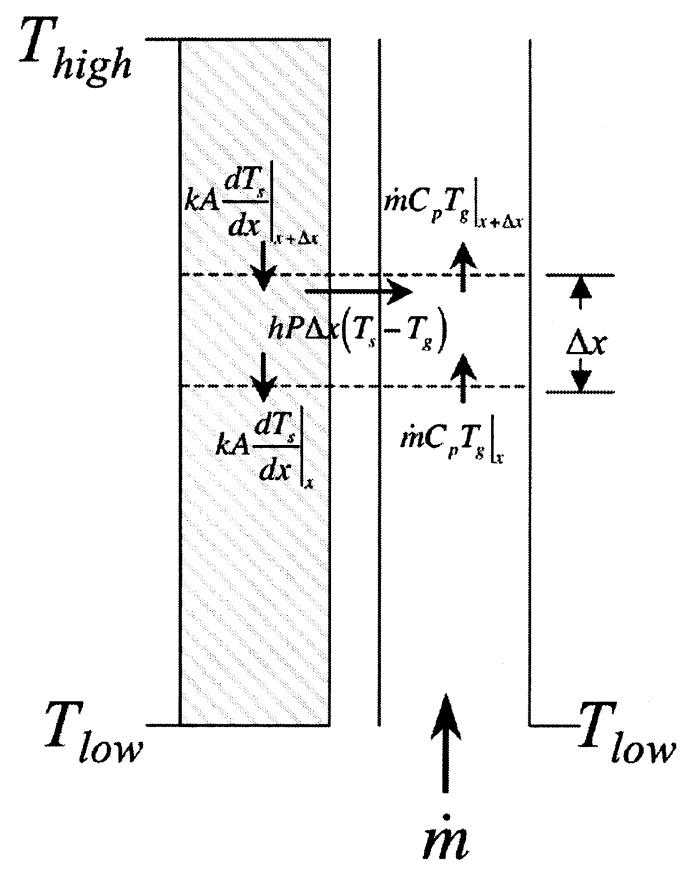

FIGURE 1. Heat balance for a one-dimensional support with continuous heat exchange to flowing gas. 
'temperature. This equation describes the simple one-dimensional fin problem in most heat transfer textbooks, except that the fluid temperature is here also a function of position. Assuming constant heat capacity, the enthalpy balance for the fluid yields

$$
\dot{m} C_{p} \frac{d T_{f}}{d x}=h P\left(T_{s}-T_{f}\right),
$$

where $\dot{m}$ is the mass flow rate of the fluid. We assume fixed boundary temperatures on the support, and a fluid temperature at the entrance fixed to the support boundary temperature:

$$
\begin{aligned}
& T_{s}(x=0)=T_{l o w}, \\
& T_{s}(x=L)=T_{\text {high }}, \text { and } \\
& T_{f}(x=0)=T_{\text {low }} .
\end{aligned}
$$

Since the problem is linear, we can set $T_{\text {low }}=0$, and normalize temperatures by $T_{\text {high }}$ : $\tau=T / T_{\text {high }}$. Replacing $x$ with the non-dimensional $\xi=x / L$ yields

$$
\begin{aligned}
& \frac{d^{2} \tau_{s}}{d \xi^{2}}=\alpha^{2}\left(\tau_{s}-\tau_{f}\right) \\
& \frac{d \tau_{f}}{d \xi}=\beta\left(\tau_{s}-\tau_{f}\right)
\end{aligned}
$$

where $\beta=\frac{h P L}{\dot{m} C_{p}}$ is the number of transfer units, and $\alpha^{2}=\frac{h P L}{k A / L}$. The solutions can be put in a reasonably compact form using the parameter $\gamma^{2}=4 \alpha^{2}+\beta^{2}$ :

$$
\begin{gathered}
\tau_{s}=\frac{(\gamma+\beta)^{2} e^{\frac{\gamma-\beta}{2} \xi}-4 \gamma \beta+(\gamma-\beta)^{2} e^{-\frac{\gamma+\beta}{2} \xi}}{(\gamma+\beta)^{2} e^{\frac{\gamma-\beta}{2}}-4 \gamma \beta+(\gamma-\beta)^{2} e^{-\frac{\gamma+\beta}{2}}} \\
\tau_{f}=2 \beta \frac{(\gamma+\beta) e^{\frac{\gamma-\beta}{2} \xi}-2 \gamma+(\gamma-\beta) e^{-\frac{\gamma+\beta}{2} \xi}}{(\gamma+\beta)^{2} e^{\frac{\gamma-\beta}{2}}-4 \gamma \beta+(\gamma-\beta)^{2} e^{-\frac{\gamma+\beta}{2}}} .
\end{gathered}
$$

The heat leak into the cold end is

$$
Q_{\text {leak }}=\left.k A \frac{d T}{d x}\right|_{x \rightarrow 0}=\left.k \frac{A}{L} T_{\text {high }} \frac{d \tau}{d \xi}\right|_{\xi \rightarrow 0} .
$$

Note that the factor $k(A / L) T_{\text {high }}$ is the heat leak down the support with no fluid flow. Thus, the non-dimensional heat leak,

$$
q_{\text {leak }}=\frac{Q_{\text {leak }}}{k \frac{A}{L} T_{\text {high }}}=\left.\frac{d \tau_{s}}{d \xi}\right|_{\xi \rightarrow 0}=\frac{\gamma\left(\gamma^{2}-\beta^{2}\right)}{(\gamma+\beta)^{2} e^{\frac{\gamma-\beta}{2}}-4 \gamma \beta+(\gamma-\beta)^{2} e^{-\frac{\gamma+\beta}{2}}} .
$$


gives the ratio of the heat with and without fluid flow, and is a function only of the parameters $\gamma$ and $\beta$, or $\alpha$ and $\beta$. It is instructive to put $q_{\text {leak }}$ in terms of a new variable, $\mu=\frac{\beta}{\alpha^{2}}=\frac{k A / L}{\dot{m} C_{p}}$, the ratio of the conductance to the capacity rate.

Figure 2 shows a contour plot of the non-dimensional heat leak as a function of $\beta$ and $\mu$. White represents a region where $q_{\text {leak }}$ is close to 1 ; that is, a region where the heat conducted down the support is affected very little by the gas flow. Black represents a region where $q_{\text {leak }}$ is close to 0 ; that is, a region where the heat conducted down the support is nearly completely suppressed by the gas flow. It is clear that when the number of transfer units, $\beta>10, q_{\text {leak }}$ depends only on $\mu$. In this case, when the support conductance is much greater than the capacity rate, $\mu>>1$, the effect of the fluid is negligible, and when the conductance is much less than the capacity flow rate, $\mu<<1$, the conductance of the tube is largely suppressed.

How does the behavior of $\beta$ and $\mu$ scale with tank size? If the radius, $R$, and length of a cylindrical support scale by the same factor, $k A / L$ depends only on thickness, $t$. Critical buckling load determines the thickness. Using the classical equation for the critical buckling load of a thin cylinder and setting it equal to the axial stress due to acceleration of the tank yields

$$
\sigma_{c r}=\frac{E}{\sqrt{3\left(1-v^{2}\right)}} \frac{t}{R}=\frac{m \ddot{x}}{2 \pi R t},
$$

where $m$ is the mass of the tank, $\ddot{x}$ is the maximum acceleration, $E$ is the Young's modulus of the support tube, and $v$ is the Poisson ratio. Since mass scales as $R^{3}, t$ scales as $R^{3 / 2}$. The capacity rate, $\dot{m} C_{p}$, is proportional to the heat load on the tank, which under most circumstances is dominated by radiation, which is in turn proportional to surface area and

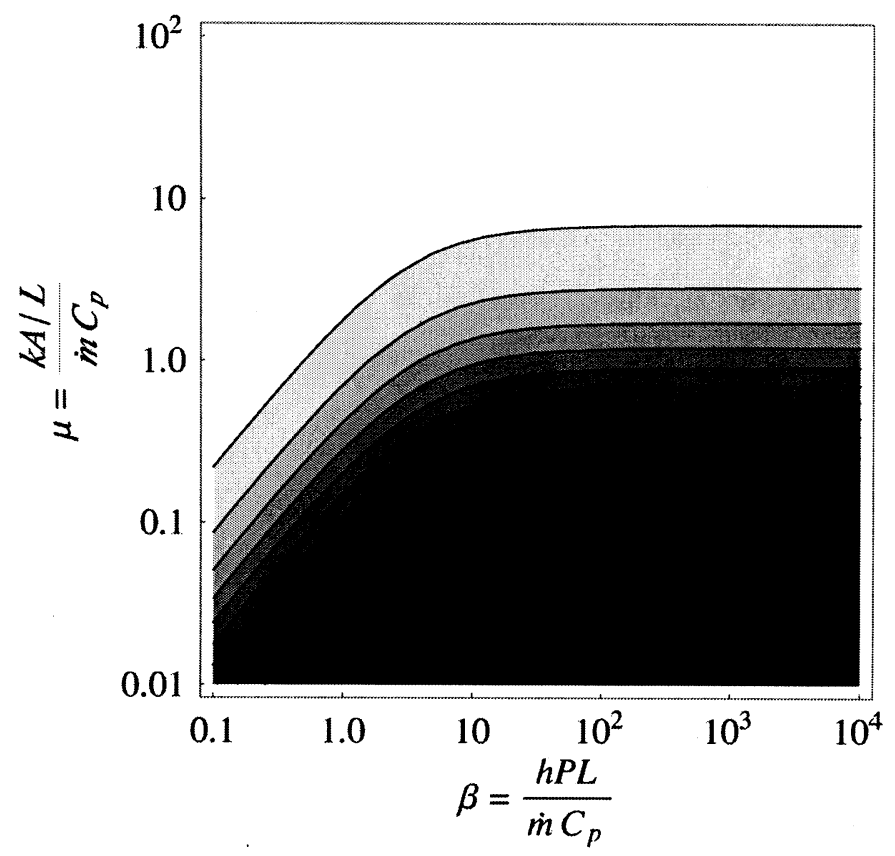

FIGURE 2. Suppression of the conductive heat leak in the one-dimensional case as a function of number of transfer units, $\beta$, and the ratio of conductance to capacity flow, $\mu$. When $\beta \gg>10$, the suppression is only a function of $\mu$. 
scales as $R^{2}$. Thus, $\mu$ scales as $R^{-1 / 2}$, and larger tanks will have lower $\mu$. For $\beta$, the product $h P$ is independent of scale for laminar flow. The length $L$ scales as $R$, so $\beta$ scales as $R^{-1}$.

\section{SUPPORT GEOMETRY}

Three types of insulating support structures are generally used for cold space flight hardware: straps, hexapod struts, and cylindrical tubes. While straps have the smallest cross-sectional area, they put the warm structure in compression, and are thus not mass efficient for large tanks. The heat conduction in a hexapod strut is determined by the requirement that the resonant frequency of the system must exceed some minimum value. For the case of a spherical tank of radius $R$ with struts attached at the radius of the tank and at the same radius on the base plate, the area-to-length ratio of each strut is given by

$$
\frac{A}{L}=\frac{4 m \omega^{2}\left(1+\zeta^{2}\right)}{3 E\left(2+5 \zeta^{2}-\sqrt{4-10 \zeta^{2}+25 \zeta^{4}}\right)},
$$

where $m$ is the mass of the tank, $\omega=2 \pi f$ is the minimum permitted resonance frequency of the horizontal mode, $E$ is the Young's modulus, and $\xi=L / R$, where $L$ is the length from the center of the base plate to the center of the tank. A similar equation can be derived for a long, slender cylinder (using beam equations), but for the more practical case of short, squat cylinders, shear dominates over bending and $A / L$ is simply given by $m \omega^{2} / G$. Approximating the shear modulus, $G=E / 2(1+v)$, the ratio of the conductances is

$$
\frac{A /\left.L\right|_{\text {strut }}}{A /\left.L\right|_{c y l}}=\frac{4\left(1+\zeta^{2}\right)}{\left(2+5 \zeta^{2}-\sqrt{4-10 \xi^{2}+25 \zeta^{4}}\right)(1+v)},
$$

where $v$ is Poisson's ratio. This function has a minimum at 2.3 (for $v=0.2$ ), so a strut support is always more than twice as conductive as a tube support with the same resonant frequency. Unfortunately, the tube thickness derived from the resonant frequency criterion is generally much too thin to withstand the local compressive stresses. Instead, thickness, $t$, is determined by the requirement that stress must remain below the critical load for local buckling, as given in equation (8). Thus, $A / L=2 \pi t / \zeta$ scales as $m^{1 / 2}$, and there is some tank mass,

$$
m^{*}=\frac{\pi \sqrt{3\left(1-v^{2}\right)} \ddot{x} E}{32 \omega^{4}} \frac{\left(2+5 \zeta^{2}-\sqrt{4-10 \zeta^{2}+25 \xi^{4}}\right)^{2}}{\left(1+\zeta^{2}\right)^{2} \zeta^{2}}
$$

below which struts give lower conductance, and above which a cylinder is superior. This analysis does not include the nonlinear correlation factor that takes into account degradation of buckling strength relative to the ideal limit due to imperfections [5], and it does not consider lateral loads, for which buckling strength is somewhat lower. However, it does only consider thin cylindrical shells; higher buckling limits can be achieved with sandwich construction or stiffeners. 


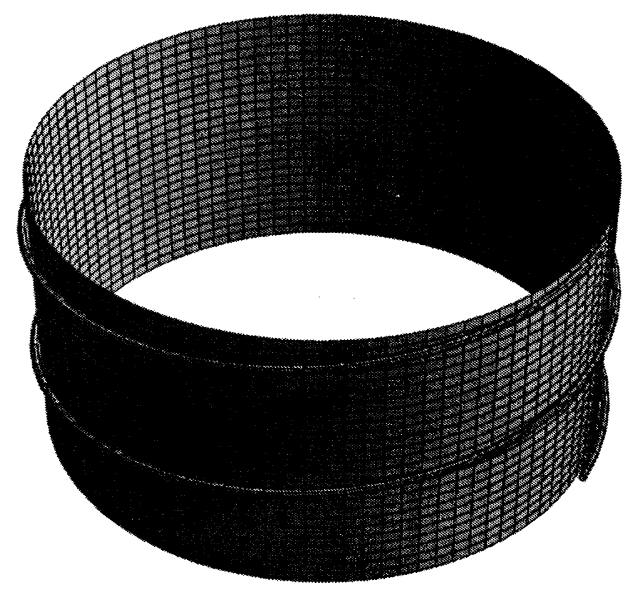

FIGURE 3. Cylindrical support cooled by helical vent tube attached continuously along its length.

\section{CYLINDER WITH HELICAL COOLING TUBE}

For a thin squat cylindrical support, the obvious geometry for intercepting heat leak by a stream of gas is with a helical tube along its surface as shown in Figure 3. Conduction in a thin cylinder can be solved by treating it as a rectangular surface with a periodic boundary condition along the edges parallel to the axis. Unfortunately, the boundary conditions for such solutions must be fixed, and cannot be a function of another variable, as in this case, where the fluid in the helical tube is described by another differential equation. Therefore, we must resort to numerical techniques. We use a standard finite difference scheme. The cylinder is broken into an array of $N_{x}$ elements in the circumferential direction and $N_{x} N_{\text {wraps }}$ in the axial direction, where $N_{\text {wraps }}$ is the number of complete turns the helical flow path makes around the cylinder. (We only treat the case where $N_{\text {wraps }}$ is an integer.) Thus, there are $N_{\text {wraps }}$ square arrays of elements and $N_{x}^{2} N_{\text {wraps }}$ equations for heat flow in the cylinder. The convective terms (equivalent to the right hand side of equation (1)) appear in the diagonal elements of each of these square arrays. The enthalpy balance equations for the fluid (equation (2)) link these $N_{x} N_{\text {wraps }}$ terms. Under the assumption of constant property values, all equations are linear, so a solution can be obtained by inverting the $\left(N_{x} N_{\text {wraps }}\left(N_{x}+1\right)\right) \times\left(N_{x} N_{\text {wraps }}\left(N_{x}+1\right)\right)$ coefficient matrix. We use the same boundary conditions as for the one-dimensional case: fixed temperatures along the hot and cold edges of the cylinder, and the inlet temperature of the gas fixed at the cold boundary temperature. For temperature dependent property values, iterating the solution works quite well, with excellent convergence being achieved in roughly 20 iterations, and no relaxation being necessary. Tests show that as $N_{x}$ increases above 50, there is little change in the solution. The algorithm was implemented with Mathematica ${ }^{\mathrm{TM}}$ on a MacIntosh Powerbook G4. A nonlinear (temperature-dependent property) solution typically took less than a minute. (Note: It was essential to use SparseArray representation, which exploits UMFPACK multifrontal direct solver methods, to achieve this performance.)

When property values are taken as independent of temperature, the non-dimensional heat leak through the cylinder, $q_{\text {leak }}$, is a function of the same two variables as in the onedimensional case, $\mu$ and $\beta$, plus two additional geometric variables, the number of wraps, $N_{\text {wraps }}$, and the cylinders aspect ratio, $\zeta$. Typically, one would not use support tubes with a very large or small aspect ratio. The examples given below all use an aspect ratio 1.6. For 

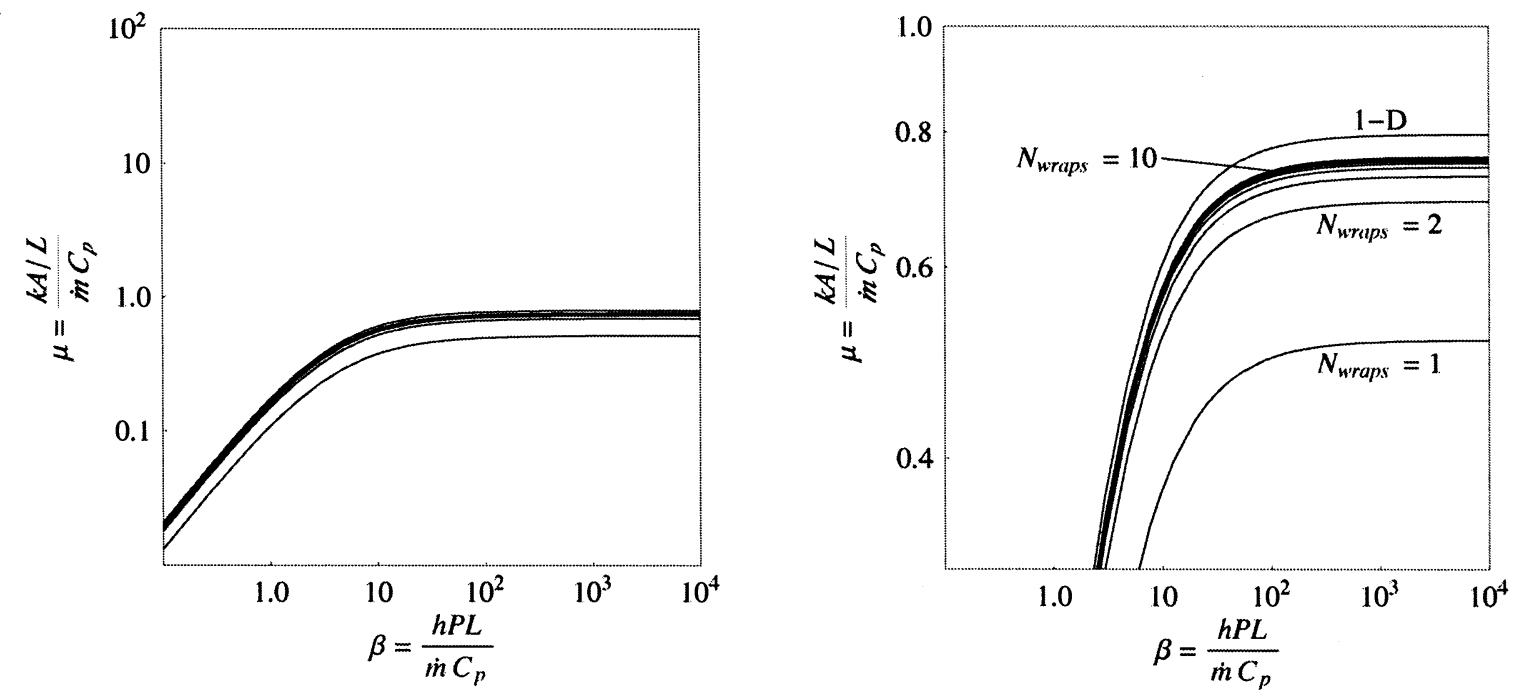

FIGURE 4. Comparison of the 0.5 contour of heat leak suppression, $q_{\text {leak }}$, determined by the inear (temperature-independent material property value) solution for $N_{\text {wraps }}=1,2, \ldots, 10$, as well as the onedimensional solution. a) Curves shown on a plot with the same range as Figure 2, for comparison. b) The same curves plotted on a narrower range of $\mu$. Note that as $N_{\text {wraps }}$ increases, it rapidly converges to a curve that is close, but not equal, to that of the one-dimensional solution.

a given value of $N_{\text {wraps }}$, we can plot a contour map of $q_{\text {leak }}$ versus $\mu$ and $\beta$, as is shown for the one-dimensional solution in Figure 2. These maps look quite similar.

To better see the differences, and to understand the effects of increasing the number of wraps of the cooling loop, we plot only the $q_{\text {leak }}=0.5$ contour for $N_{\text {wraps }}=1,2 \ldots, 10$ in Figure 4, along with the $q_{\text {leak }}=0.5$ contour from the one-dimensional solution. The figure shows a significant improvement in performance in going from one to two wraps. That is, for the same thermal conductance to capacity flow rate, two wraps produces a greater suppression of the heat leak than one. However, beyond two wraps, the contours quickly converge. Interestingly, it converges to a line close, but not equal to, the one-dimensional case. In the region $\beta \gg>10$, where $q_{\text {leak }}$ becomes independent of $\beta, q_{\text {leak }}=0.5$ occurs at $\mu=$ 0.76 for the cylinder, but $\mu=0.80$ for the one-dimensional solution. In general, the onedimensional solution is a very good approximation to the two-dimensional numerical solution for $N_{\text {wraps }} \geq 2$. Note that numerical solution treats the number of transfer units, $\beta=\frac{h P L}{\dot{m} C_{p}}$, and $N_{\text {wraps }}$ as independent variables, but for a constant pipe cross section, $h P L$ will increase with the number of wraps. However, $h P$ can often be made large with clever internal geometry. As noted above, if $\beta>>10$, the solution is nearly independent of $\beta$.

The temperature-dependent properties solution is not linear in temperature, so it is not a function of a few simple non-dimensional variables. A typical solution is shown in Figure 5. For this case, the support tube was $\mathrm{G} 10$ with a radius of $406 \mathrm{~mm}$, a length of 640 $\mathrm{mm}$, and a thickness of $1 \mathrm{~mm}$. The number of wraps is 2 , and the boundary conditions were $T_{\text {high }}=100 \mathrm{~K}$ and $T_{\text {low }}=24.6 \mathrm{~K}$. The cooling gas was hydrogen flowing at a rate of $0.69 \mathrm{mg} / \mathrm{s}$. This flow rate was sufficient to strongly affect the temperature profile: the thermal conductivity of G10, as that of most materials, increases with increasing temperature, producing a temperature profile that is flat at the high temperature end, and decreases more sharply at the low end. The flow reverses this curvature, producing a profile that has a lower gradient, and thus lower heat flow, at the cold end. The calculated heat leak into the cold end is $13.8 \mathrm{~mW}$, which is $13.5 \%$ of the heat leak with no gas flow. 


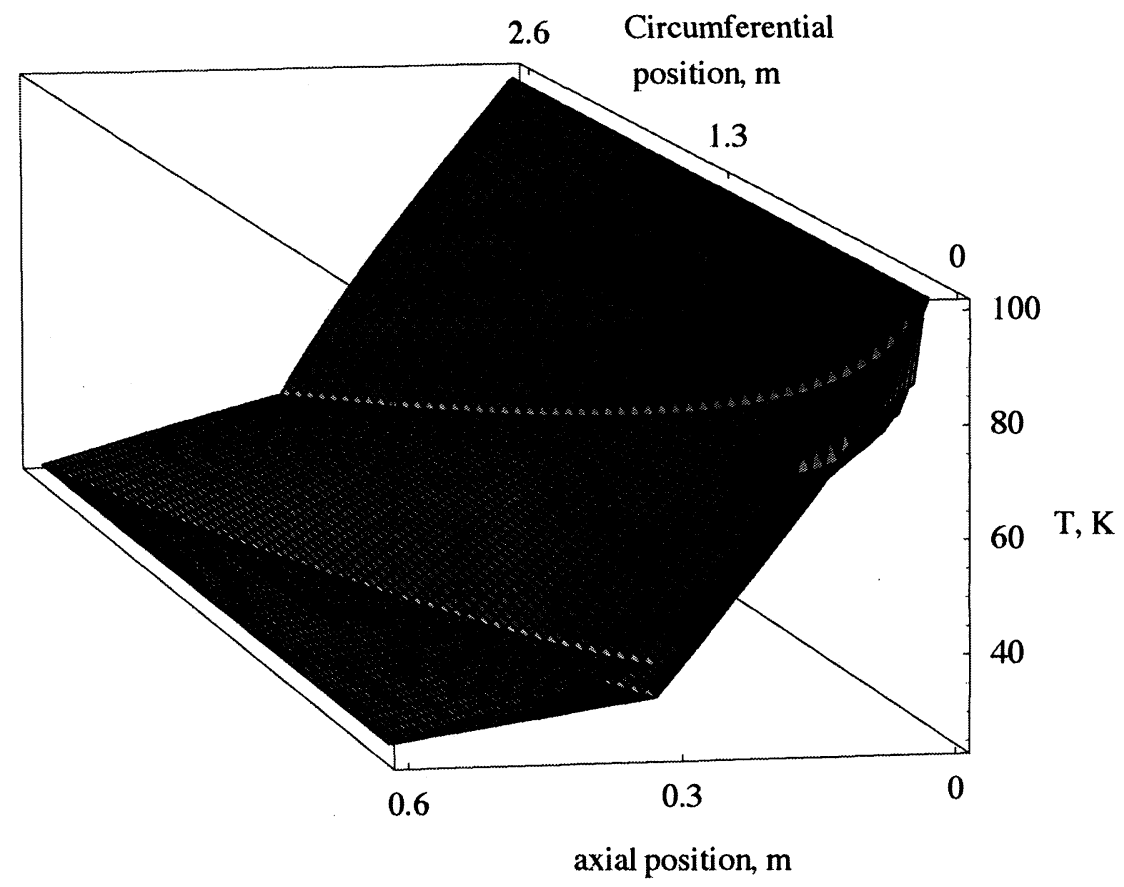

FIGURE 5. Temperature as a function of position on the cylinder. Top surface - nonlinear (temperature dependent material property value) solution. Bottom surface - linear (temperature independent material property value) solution. The linear solution is everywhere lower than the nonlinear, except at the upper and lower edges.

Also shown in the figure is the temperature-independent properties solution. In this example, the temperature independent solution is everywhere lower, and it generates a significantly lower heat value, $10.9 \mathrm{~mW}$, or $10.6 \%$ of the heat leak with no flow. (The average thermal conductivity is chosen so that the heat leak with no flow is the same in the linear and non-linear solutions.) There are some situations where the linear (temperatureindependent properties) solution gives a higher heat leak value than the nonlinear, although the computed heat leaks are close to those computed from the full nonlinear solution.

\section{CONCLUSIONS}

For supporting large cryogen tanks against severe launch loads, a cylindrical support is superior to a hexapod strut above a certain size. Reducing the heat flow into the tank can be accomplished by attaching the vent line in a helix along the surface of the cylinder, so that the boil-off gas intercepts the inflowing heat. Modeling of this geometry shows that a helix of two wraps effectively intercepts the heat, and that with two or more wraps, the results match well to a simple one-dimensional analytic solution. Including the temperature dependence of the material property values has a significant, but not overwhelming, effect on the computed heat leak.

\section{REFERENCE}

1. Bejan, A. and Smith, J. L., Jr., Cryogenics 14, pp.158-163 (1974).

2. Bejan, A., Cryogenics 15 pp.290-292 (1975).

3. Chato, J. C. and Khodadi, J. M., J. Heat Transfer 106, pp. 871-875 (1984).

4. Hofmann, A., Cryogenics, 44, pp. 159-165 (2004).

5 Weingarten, V.I., et al., NASA SP-8007: Buckling of Thin-Walled Circular Cylinders, NASA (1968). 